\title{
QUASI-MULTIPLICATIVE MAPS ON BAER SEMIGROUPS
}

\author{
by C. S. JOHNSON, Jr*
}

(Received 30 June, 1970)

1. Introduction. A Baer semigroup is a semigroup $S$ with 0 and 1 in which, for each $x \in S$, the left annihilator $L(x)=\{y \in S: y x=0\}$ of $x$ is a principal left ideal generated by an idempotent and the right annihilator $R(x)=\{y \in S: x y=0\}$ of $x$ is a principal right ideal generated by an idempotent. Baer semigroups are of interest because (see [5]) the left annihilators of the elements of a Baer semigroup $S, \mathscr{L}(S)=\{L(x): x \in S\}$, form a bounded lattice and (see [4]) every bounded lattice arises in this manner. In this note we look at a type of map $\phi$ on a Baer semigroup $S$ which has the property that $S \phi$ is a Baer semigroup. (The homomorphic image of a Baer semigroup need not be a Baer semigroup. For a case where it is, see [7].) When the Baer semigroup is specialized to a Boolean algebra, this type of map generalizes Halmos's notion of a quantifier.

\section{Quasi-multiplicative maps.}

Definition 1. A map $\phi$ of a semigroup $S$ into itself is quasi-multiplicative if $((x \phi) y) \phi=$ $(x(y \phi)) \phi=(x \phi)(y \phi)$ for all $x, y \in S$.

THEOREM 2. If $S$ is a Baer semigroup and $\phi: S \rightarrow S$ is a zero-preserving, idempotent, quasi-multiplicative map, then $S \phi$ is a subsemigroup of $S$ and a Baer semigroup in its own right.

Proof. It is clear that $S \phi$ is a subsemigroup of $S$. If $x \in S$, let $(x \phi)^{l}$ be the idempotent that generates $L(x \phi)$, i.e., $L(x \phi)=S(x \phi)^{l}$. We claim that, in $S \phi$, we have $L(x \phi)=(S \phi)\left((x \phi)^{l} \phi\right)$, and that $(x \phi)^{l} \phi$ is an idempotent. From $\left((x \phi)^{l} \phi\right)(x \phi)=\left((x \phi)^{l} x \phi\right) \phi=0 \phi=0$ it follows that $(S \phi)\left((x \phi)^{l} \phi\right) \subseteq L(x \phi)$. It also follows that $(x \phi)^{l} \phi$ is idempotent, since $(y \phi)(x \phi)=0 \Rightarrow y \phi=$ $(y \phi)(x \phi)^{l} \Rightarrow y \phi=y \phi \phi=\left((y \phi)(x \phi)^{l}\right) \phi=(y \phi)\left((x \phi)^{l} \phi\right)$. This last argument also shows that, in $S \phi, L(x \phi) \subseteq(S \phi)\left((x \phi)^{l} \phi\right)$. A dual argument shows that, in $S \phi$, we have $R(x \phi)=\left((x \phi)^{r} \phi\right)(S \phi)$, where $(x \phi)^{r}$ is the idempotent that generates $R(x \phi)$ in $S$, and that $(x \phi)^{r} \phi$ is an idempotent.

We have as a corollary the following result from [1].

COROLlaRy 3. If $S$ is a Baer semigroup and $e=e^{2} \in S$, then eSe is a Baer semigroup.

Proof. The map $x \mapsto e x e$ is idempotent, zero-preserving and quasi-multiplicative.

REMARK. To see that a zero-preserving, idempotent, quasi-multiplicative map $\phi$ on a Baer semigroup $S$ need not be of the form $x \phi=(1 \phi) x(1 \phi)$, notice that any map $\phi: S \rightarrow S$ such that range $\phi=\{0,1\}, 0 \phi=0$, and $1 \phi=1$ is idempotent and quasi-multiplicative.

THEOREM 4. Any quasi-multiplicative map that preserves 1 is idempotent. If $\phi$ is any zero-preserving, idempotent, quasi-multiplicative map on a Baer semigroup $S$, then $\phi=\theta \psi$,

\footnotetext{
* The results presented here were part of a thesis submitted to the University of Massachusetts. The research was supported by an NSF Traineeship.

B
} 
where there is an idempotent $e \in S$ such that $x \theta=$ exe and where $\psi$, considered as a map on $S \theta=e S e$, is zero-preserving, quasi-multiplicative and 1-preserving.

Proof. To see the first part, notice that $(x \phi) \phi=((x \phi) 1) \phi=(x \phi)(1 \phi)=(x \phi) 1=x \phi$. For the second assertion let $e=1 \phi$. We have $(1 \phi)(1 \phi)=((1 \phi) 1) \phi=1 \phi \phi=1 \phi$. Now let $\psi=\left.\phi\right|_{e S e} . \psi$ clearly preserves $1 \phi$, which is the unit element of $e S e$. Finally, $((1 \phi) x(1 \phi)) \phi=$ $(1 \phi)(\mathrm{x}(1 \phi)) \phi=(1 \phi)((\mathrm{x} \phi) 1) \phi=\left(1^{\prime} \phi\right)(x \phi)=(1(x \phi)) \phi=x \phi \phi=x \phi$, which shows that $\phi=\theta \psi$ when $x \theta=$ exe.

REMARK. It is shown in [1] that $\mathscr{L}(e S e)$ is isomorphic to the set of fixed points of a certain map on $\mathscr{L}(S)$.

3. Quasi-multiplicative residuated maps on a Boolean algebra. A Boolean algebra, considered as a semigroup with respect to the meet operation, is a Baer semigroup. In this lattice context the condition in Definition 1 becomes $(x \wedge y \phi) \phi=x \phi \wedge y \phi$. A map $\phi$ on a Boolean algebra $L$ which has this property and which is zero-preserving and increasing ( $x \leqq x \phi$ for all $x \in L$ ) is what Halmos calls [3, p. 220] a quantifier. Quantifiers were studied in the more general context of an orthomodular lattice by M. F. Janowitz in [6]. It turns out that quantifiers are residuated maps and that there is a notion of adjoint available for residuated maps on a Boolean algebra (or any other involution partially ordered set). On using this adjoint, it follows from Theorem 3 of [3] that the quantifiers on a Boolean algebra are precisely the increasing projections ( $\phi$ being a projection if $\phi=\phi^{2}=\phi^{*}$ ). We shall use this adjoint to characterize quasi-multiplicative residuated maps on a Boolean algebra.

A map $\phi$ of a lattice $L$ into itself is residuated if the inverse image of a principal ideal is again a principal ideal or, equivalently, if $\phi$ is isotone and there is another isotone map $\phi^{+}$ (called a residual map) of $L$ into itself such that $x \phi^{+} \phi \leqq x \leqq x \phi \phi^{+}$for all $x \in L$. Residuated maps are join homomorphisms and preserve the lattice zero if it has one. The semigroup of residuated maps on a lattice $L$ will be denoted by $S(L)$. Under the pointwise partial order, $S(L)$ is a join semilattice with, for $\phi, \psi \in S(L), \phi \vee \psi$ and $(\phi \vee \psi)^{+}$given by $x(\phi \vee \psi)=x \phi \vee x \psi$ and $x(\phi \vee \psi)^{+}=x \phi^{+} \wedge x \psi^{+}$.

LEMMA 5. If $L$ is a Boolean algebra and $\phi \in S(L)$, then $\phi^{*}$, given by $x \phi^{*}=\left(x^{\prime} \phi^{+}\right)^{\prime}$ for all $x \in L$, is residuated and the map $\phi \rightarrow \phi^{*}$ is a semigroup involution on $S(L)$.

Proof. This follows from Lemma 22 of [5].

Lemma 6. Let $L$ be a Boolean algebra. An isotone map $\phi: L \rightarrow L$ is residuated if and only if there exists an isotone map $\psi: L \rightarrow L$ such that, for each $x \in L$,

(i) $(x \phi)^{\prime} \psi \leqq x^{\prime}$,

(ii) $(x \psi)^{\prime} \phi \leqq x^{\prime}$.

If such a $\psi$ exists, it is unique and equals $\phi^{*}$.

Proof. This follows from a proof on p. 651 of [2].

CoRollary 7. If $L$ is a Boolean algebra and $\phi, \psi \in S(L)$, then $(\phi \vee \psi)^{*}=\phi^{*} \vee \psi^{*}$. 
Proof. $(x(\phi \vee \psi))^{\prime}\left(\phi^{*} \vee \psi^{*}\right)=\left(x \phi^{\prime} \wedge x \psi^{\prime}\right)\left(\phi^{*} \vee \psi^{*}\right)=\left(x \phi^{\prime} \wedge x \psi^{\prime}\right) \phi^{*} \vee\left(x \phi^{\prime} \wedge x \psi^{\prime}\right) \psi^{*} \leqq$ $x \phi^{\prime} \phi^{*} \vee x \psi^{\prime} \psi^{*} \leqq x^{\prime}$ and similarly $\left(x\left(\phi^{*} \vee \psi^{*}\right)\right)^{\prime}(\phi \vee \psi) \leqq x^{\prime}$.

LEMMA 8. A quasi-multiplicative residuated map $\phi$ on a lattice $L$ is zero-preserving (if $L$ has a zero) and is idempotent.

Proof. Since $\phi$ is residuated, it preserves the zero and joins; $\phi$ is idempotent since $x \phi \phi=(x \phi \wedge(x \vee x \phi)) \phi=x \phi \wedge(x \vee x \phi) \phi=x \phi \wedge(x \phi \vee x \phi \phi)=x \phi$.

LEMMA 9. If $L$ is a Boolean algebra and $\phi \in S(L)$, then $\phi$ is quasi-multiplicative if $\phi^{2} \leqq \phi$ and $\phi \phi^{*} \leqq \phi$.

Proof. Notice first that $y \phi \phi^{*} \leqq y \phi \Rightarrow\left((y \phi)^{\prime} \phi^{+}\right)^{\prime} \leqq y \phi \Rightarrow(y \phi)^{\prime} \leqq(y \phi)^{\prime} \phi^{+} \Rightarrow(y \phi)^{\prime} \phi \leqq$ $(y \phi)^{\prime} \Rightarrow y \phi \leqq\left((y \phi)^{\prime} \phi\right)^{\prime}$. Notice also that, since $x \phi \leqq\left(x \vee(y \phi)^{\prime}\right) \phi=\left[(x \wedge y \phi) \vee(y \phi)^{\prime}\right] \phi=$ $(x \wedge y \phi) \phi \vee(y \phi)^{\prime} \phi$, we have $x \phi \wedge\left((y \phi)^{\prime} \phi\right)^{\prime} \leqq(x \wedge y \phi) \phi$. Now $x \phi \wedge y \phi \leqq x \phi \wedge\left((y \phi)^{\prime} \phi\right)^{\prime} \leqq$ $(x \wedge y \phi) \phi$. On the other hand, $(x \wedge y \phi) \phi \leqq x \phi$ and $(x \wedge y \phi) \phi \leqq y \phi \phi \leqq y \phi$; so $(x \wedge y \phi) \phi \leqq$ $x \phi \wedge y \phi$. This shows that $(x \wedge y \phi) \phi=x \phi \wedge y \phi$.

LEMMA 10. If $L$ is a Boolean algebra and $\phi \in S(L)$ is quasi-multiplicative, then $\psi \in S(L)$, given by $x \psi=x \phi \wedge\left(0 \phi^{+}\right)^{\prime}$, is a projection.

Proof. $\psi \in S(L)$ since it is the composition of two residuated maps. (In a Boolean algebra $x \mapsto x \wedge e$ is residuated with $x \mapsto x \vee e^{\prime}$ as its residual.) To show that $\psi=\psi^{*}$ it will suffice, by Lemma 6 , to show that $(x \psi)^{\prime} \psi \leqq x^{\prime}$ for all $x \in L$. Now $(x \psi)^{\prime} \psi=\left(x \phi \wedge\left(0 \phi^{+}\right)^{\prime}\right)^{\prime} \phi \wedge\left(0 \phi^{+}\right)^{\prime}=$ $\left((x \phi)^{\prime} \vee 0 \phi^{+}\right) \phi \wedge\left(0 \phi^{+}\right)^{\prime}=(x \phi)^{\prime} \phi \wedge\left(0 \phi^{+}\right)^{\prime} ; \quad$ so we have $(x \psi)^{\prime} \psi \leqq x^{\prime} \Leftrightarrow(x \phi)^{\prime} \phi \wedge\left(0 \phi^{+}\right)^{\prime} \leqq$ $x^{\prime} \Leftrightarrow(x \phi)^{\prime} \phi \wedge\left(0 \phi^{+}\right)^{\prime} \wedge x=0 \Leftrightarrow(x \phi)^{\prime} \phi \wedge x \leqq 0 \phi^{+} \Leftrightarrow\left((x \phi)^{\prime} \phi \wedge x\right) \phi=0$. But $\left((x \phi)^{\prime} \phi \wedge x\right) \phi=$ $(\mathrm{x} \phi)^{\prime} \phi \wedge x \phi=\left((x \phi)^{\prime} \wedge x \phi\right) \phi=0 \phi=0$; so we have $\psi=\psi^{*}$. To show that $\psi^{2}=\psi$ it will be sufficient to show that $\psi^{2} \leqq \psi$, for then we shall have $\psi \psi^{*}=\psi^{2} \leqq \psi$, making $\psi$ quasi-multiplicative by Lemma 9 and hence idempotent by Lemma 8. But $x \psi \psi=\left(x \phi \wedge\left(0 \phi^{+}\right)^{\prime}\right) \phi \wedge\left(0 \phi^{+}\right)^{\prime}$ $\leqq x \phi \phi \wedge\left(0 \phi^{+}\right)^{\prime}=x \phi \wedge\left(0 \phi^{+}\right)^{\prime}=x \psi$. This completes the proof.

REMARK. The converse of Lemma 10 is false. Consider an 8-element Boolean algebra with atoms $a, b$, and $c$. The map $\psi_{a}$ given by

is residuated with $\psi_{a}^{+}$given by

$$
x \psi_{a}=\left\{\begin{array}{ccc}
0 & \text { if } & x \leqq a, \\
x \vee a & \text { if } & x \leqq a
\end{array}\right\}
$$

$$
x \psi_{a}^{+}=\left\{\begin{array}{lll}
x & \text { if } & a \leqq x \\
a & \text { if } & a \leq x
\end{array}\right\}
$$

The map $\psi_{a}$ is not quasi-multiplicative, since $\left(c^{\prime} \wedge b^{\prime} \psi_{a}\right) \psi_{a}=a \psi_{a}=0$, whereas $c^{\prime} \psi_{a} \wedge b^{\prime} \psi_{a}=$ $c^{\prime} \wedge b^{\prime}=a$. However,

$$
x \psi_{a} \wedge\left(0 \psi_{a}^{+}\right)^{\prime}=x \psi_{a} \wedge a^{\prime}=\left\{\begin{array}{ccc}
0 \wedge a^{\prime} & \text { if } & x \leqq a \\
(x \vee a) \wedge a^{\prime} & \text { if } & x \leqq a
\end{array}\right\}=\left\{\begin{array}{lll}
x \wedge a^{\prime} & \text { if } & x \leqq a, \\
x \wedge a^{\prime} & \text { if } & x \leqq a
\end{array}\right\}=x \wedge a^{\prime} ;
$$

so $\psi$ is a projection. 
THEOREM 11. Let $L$ be a Boolean algebra and $\phi \in S(L)$. The following are equivalent.

(a) $\phi$ is quasi-multiplicative.

(b) $\phi=\phi_{1} \vee \phi_{2}$, where $\phi_{1}, \phi_{2} \in S(L), \phi_{1}$ is a projection, $\phi_{1} \phi_{2}=\phi_{2}, \phi_{2} \phi_{1}=0$, and $\phi_{2} \phi_{2}^{*} \leqq \phi_{1}$.

(c) $\phi^{2} \leqq \phi$ and $\phi \phi^{*} \leqq \phi$.

Proof. (a) $\Rightarrow$ (b). Let $\phi_{1}$ and $\phi_{2}$ be given by $x \phi_{1}=x \phi \wedge\left(0 \phi^{+}\right)^{\prime}$ and $x \phi_{2}=x \phi \wedge 0 \phi^{+}$. It is clear that $x \phi=x \phi_{1} \vee x \phi_{2}$ for all $x \in L ; \phi_{1}, \phi_{2} \in S(L)$ since they are compositions of residuated maps. $\phi_{1}$ is a projection by Lemma 10 .

$\phi_{1} \phi_{2}=\phi_{2}$ since $x \phi_{2}=x \phi \wedge 0 \phi^{+}=(x \phi) \phi \wedge 0 \phi^{+}=\left[\left(x \phi \wedge 0 \phi^{+}\right) \vee\left(x \phi \wedge\left(0 \phi^{+}\right)^{\prime}\right)\right] \phi \wedge 0 \phi^{+}$ $=\left[\left(x \phi \wedge 0 \phi^{+}\right) \phi \vee\left(x \phi \wedge\left(0 \phi^{+}\right)^{\prime}\right) \phi\right] \wedge 0 \phi^{+}=\left(x \phi \wedge\left(0 \phi^{+}\right)^{\prime}\right) \phi \wedge 0 \phi^{+}=x \phi_{1} \phi_{2}$ for all $x \in L$.

$\phi_{2} \phi_{1}=0$ since $x \phi_{2} \phi_{1}=\left(x \phi \wedge 0 \phi^{+}\right) \phi \wedge\left(0 \phi^{+}\right)^{\prime} \leqq 0 \phi^{+} \phi=0$ for all $x \in L$.

Notice that $\phi_{2}^{+}$is given by $x \phi_{2}^{+}=\left(x \vee\left(0 \phi^{+}\right)^{\prime}\right) \phi^{+}$. Thus $x \phi_{2} \phi_{2}^{*}=\left(x \phi \wedge 0 \phi^{+}\right) \phi_{2}^{*}=$ $\left(\left(x \phi \wedge 0 \phi^{+}\right)^{\prime} \phi_{2}^{+}\right)^{\prime}=\left(\left((x \phi)^{\prime} \vee\left(0 \phi^{+}\right)^{\prime}\right) \phi_{2}^{+}\right)^{\prime}=\left(\left((x \phi)^{\prime} \vee\left(0 \phi^{+}\right)^{\prime}\right) \phi^{+}\right)^{\prime}$. We thus have $x \phi_{2} \phi_{2}^{*} \leqq$ $\left(\left(0 \phi^{+}\right)^{\prime} \phi^{+}\right)^{\prime}$ and $x \phi_{2} \phi_{2}^{*} \leqq\left((x \phi)^{\prime} \phi^{+}\right)^{\prime}$. Now $0 \phi^{+} \phi=0 \leqq\left(0 \phi^{+}\right)^{\prime} \Rightarrow 0 \phi^{+} \leqq\left(0 \phi^{+}\right)^{\prime} \phi^{+} \Rightarrow$ $\left(\left(0 \phi^{+}\right)^{\prime} \phi^{+}\right)^{\prime} \leqq\left(0 \phi^{+}\right)^{\prime} \Rightarrow x \phi_{2} \phi_{2}^{*} \leqq\left(0 \phi^{+}\right)^{\prime}$. In addition, $(x \phi)^{\prime} \phi \wedge x \phi=\left((x \phi)^{\prime} \wedge x \phi\right) \phi=0 \Rightarrow$ $(x \phi)^{\prime} \phi \leqq(x \phi)^{\prime} \Rightarrow(x \phi)^{\prime} \leqq(x \phi)^{\prime} \phi^{+} \Rightarrow\left((x \phi)^{\prime} \phi^{+}\right)^{\prime} \leqq x \phi \Rightarrow x \phi_{2} \phi_{2}^{*} \leqq x \phi . \quad$ This shows that $x \phi_{2} \phi_{2}^{*} \leqq x \phi \wedge\left(0 \phi^{+}\right)^{\prime}=x \phi_{1}$ for all $x \in L$, and so $\phi_{2} \phi_{2}^{*} \leqq \phi_{1}$.

(b) $\Rightarrow$ (c). Suppose that $\phi=\phi_{1} \vee \phi_{2}$, where $\phi_{1}$ and $\phi_{2}$ are as in (b). Then $x \phi^{2}=$ $\left(x \phi_{1} \vee x \phi_{2}\right)\left(\phi_{1} \vee \phi_{2}\right)=x \phi_{1} \phi_{1} \vee x \phi_{2} \phi_{1} \vee x \phi_{1} \phi_{2} \vee x \phi_{2} \phi_{2}$. Since $\phi_{1} \phi_{1}=\phi_{1}, \phi_{2} \phi_{1}=0$, $\phi_{1} \phi_{2}=\phi_{2}$ and $\phi_{2} \phi_{2}=\phi_{2} \phi_{1} \phi_{2}=0$, we have $x \phi^{2}=x \phi_{1} \vee x \phi_{2}=x \phi$. By Corollary 7 we have that $\left(\phi_{1} \vee \phi_{2}\right)^{*}=\phi_{1}^{*} \vee \phi_{2}^{*}$. Thus $x \phi \phi^{*}=\left(x \phi_{1} \vee x \phi_{2}\right)\left(\phi_{1}^{*} \vee \phi_{2}^{*}\right)=x \phi_{1} \phi_{1}^{*} \vee x \phi_{2} \phi_{1}^{*} \vee$ $x \phi_{1} \phi_{2}^{*} \vee x \phi_{2} \phi_{2}^{*}$. Since $\phi_{1} \phi_{1}^{*}=\phi_{1}, \phi_{2} \phi_{1}^{*}=\phi_{2} \phi_{1}=0, \phi_{1} \phi_{2}^{*}=\left(\phi_{2} \phi_{1}^{*}\right)^{*}=\left(\phi_{2} \phi_{1}\right)^{*}=$ $0^{*}=0$, and $\phi_{2} \phi_{2}^{*} \leqq \phi_{1}$, we have $x \phi \phi^{*} \leqq x \phi_{1} \leqq x \phi_{1} \vee x \phi_{2}=x \phi$. Thus $\phi \phi^{*} \leqq \phi$.

(c) $\Rightarrow$ (a). This is Lemma 9.

The author is grateful to Professor M. F. Janowitz for his generous help and guidance.

\section{REFERENCES}

1. T. S. Blyth and M. F. Janowitz, On decreasing Baer semigroups, Bull. Soc. Roy. Sci, Liège 38 (1969), 414-423.

2. D. J. Foulis, Baer *-semigroups, Proc. Amer. Math. Soc. 11 (1960), 648-654. 249.

3. P. R. Halmos, Algebraic logic I, monadic Boolean algebras, Compositio Math. 12 (1955), 217-

4. M. F. Janowitz, A semigroup approach to lattices, Canad. J. Math. 18 (1966), 1212-1223.

5. M. F. Janowitz, Baer semigroups, Duke Math. J. 32 (1965), 85-95.

6. M. F. Janowitz, Quantifiers and orthomodular lattices, Pacific J. Math. 13 (1963), 1241-1249.

7. B. J. Thorne, A-P congruences on Baer semigroups, Pacific J. Math. 28 (1969), 681-698.

UNIVERSITY OF MASSACHUSETTS

AMHERST, MASSACHUSETTS 01002 